\title{
Las sociedades de beneficio e interés colectivo en el Perú: un análisis de legislación comparada en Iberoamérica y Norteamérica
}

\section{Collective benefit and interest companies as a vehicle for sustainability: a comparative perspective}

\author{
Edgar Romario Aranibar Ramos \\ Universidade de São Paulo, Brasil•romario.aranibar@usp.br \\ Fabiola Choque Zambrano \\ Universidad Nacional de San Agustín de Arequipa, Perú • fchoquez@unsa.edu.pe \\ Antony Jonny Patiño Huayhua \\ Universidad Nacional de San Agustín de Arequipa, Perú • apatinoh@unsa.edu.pe
}

\section{Resumen}

Las crisis en el plano internacional han conllevado que las empresas sean más conscientes de su influencia en las cuestiones globales, así como de las medidas a aplicar; de esta forma, han generado un asidero para la adopción pública y privada de los Objetivos de Desarrollo Sostenible (ODS) propuestos en la Agenda 2030. El presente trabajo analiza el desarrollo de las empresas o sociedades de beneficio e interés colectivo (BIC) en Perú, bajo una óptica de legislación y administración comparada con países de Iberoamérica y América del Norte: España, Colombia, Ecuador, Argentina, Chile, México, Uruguay, El Salvador, Estados Unidos de América y Canadá. Para ello, se recurre a una revisión bibliográfica en repositorios gubernamentales y académicos. De esta manera, se identifican los avances jurídicos, refrendados en leyes, al igual que un impulso de la iniciativa privada, para la construcción de este modelo enfocado en el desarrollo sostenible incorporado en la constitución de la empresa societaria. Por otro lado, también se reconocen complicaciones en la transición de una empresa a una empresa societaria BIC, puesto que en varios países no implica ningún beneficio directo para estas organizaciones y, en cambio, implica el direccionamiento presupuestario, presentación de informes y transparencia plena en aras de conservar la figura jurídica.

illustro• Universidad Católica San Pablo, Arequipa • Vol. 12, 2021, 120-137• e-ISSN 2710-2440

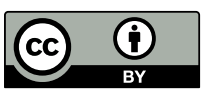


Palabras clave: sociedades de beneficio e interés colectivo (BIC), empresas B, Objetivos de Desarrollo Sostenible (ODS), triple impacto, Ley N ${ }^{\circ} 31072$ Ley de la Sociedad de Beneficio e Interés Colectivo.

\begin{abstract}
International crises have led companies to be more aware about their influence in global issues, as well as remedial measures to apply; thus, a base has been provided for the adoption, in public and private sectors, of the Sustainable Development Goals (SDG), proposed in Agenda 2030. The present work analyzes the development of collective benefit and interest companies (BIC), in Peru, from a perspective of legislation and administration compared to Iberoamerican and North American countries: Spain, Colombia, Ecuador, Argentina, Chile, Mexico, Uruguay, El Salvador, the United States of America and Canada. For this purpose, a bibliographic review in governmental and academic repositories was carried out. Legal advances, endorsed in laws, are identified, as well as an impulse from the private initiative, for the construction of this model focused on sustainable development incorporated in the constitution of the corporate company. Also, complications during transition from a company or a B company to a BIC are recognized; in particular, various countries do not offer any direct benefit for these organizations and, instead, it involves budget management, the presentation of reports and full transparency in order to preserve the legal status.
\end{abstract}

Keywords: collective benefit and interest companies (BIC), B-Corp, Sustainable Development Goals (SDG), triple impact, Law N 31072 Law of the Benefit and Collective Interest Society.

\title{
1. Introducción
}

La Comisión Mundial sobre Medio Ambiente y Desarrollo de la Organización de Naciones Unidas presentó el término «desarrollo sostenible» como la capacidad de satisfacer las necesidades de las generaciones presentes, sin comprometer la capacidad de satisfacción de necesidades de las generaciones futuras (Emas, 2015). En la actualidad, los Objetivos de Desarrollo Sostenible (ODS) se han introducido tanto en el sector público como privado, en concordancia con una nueva gama de expectativas sobre el comportamiento y postura de las organizaciones frente a la sustentabilidad social y medioambiental de sus operaciones (Bajo, González y Fernández, 2013).

Según Baltazar Caravedo (2017), la evolución del propósito de las personas jurídicas con fines de lucro se puede dividir en tres momentos cronológicos. En primer lugar se encuentran las empresas tradicionales, cuya finalidad principal es la maximización de utilidades; en segundo lugar, las empresas de responsabilidad social, las cuales también tienen como finalidad principal la maximización de utilidades junto con la consideración de invertir en problemas sociales y medioambientales vinculados a su actividad económica; y, finalmente, las empresas con propósito, las cuales a diferencia de las antes descritas, tienen como finalidad la generación de utilidades conjuntamente con 
un propósito concreto de resolver un problema social y/o medioambiental (donde se enmarcan las empresas B y las sociedades BIC).

$\mathrm{Al}$ respecto, es importante reconocer y diferenciar una sociedad BIC de una empresa B, dado que por su nomenclatura y algunos rasgos pueden confundirse. Una sociedad BIC cuenta con el reconocimiento legal de un gobierno; es un modelo jurídico, y no es necesaria su certificación como empresa B (Abramovay, 2013). En cambio, una empresa B supone la obtención de una certificación corporativa otorgada por B Lab (persona jurídica sin fines de lucro cofundada en 2006 por Jay Coen Gilbert en Estados Unidos de América), Sistema B (Sistema B, 2020) o alguna otra certificadora. De este modo, el certificado de empresa B implica una rigurosa evaluación internacional, que tiene como objetivo el reconocimiento y promoción de organizaciones que cumplen con estándares de transparencia, desempeño, sustentabilidad y responsabilidad social (Salmeron, 2019). Debido al enfoque que muestran sobre la sostenibilidad, tanto las empresas BIC como las empresas B son reconocidas como empresas de «triple impacto». El término «triple impacto» hace referencia a un modelo económico que trasciende a la rentabilidad financiera; busca generar un impacto social y medioambiental positivo (Aimar, Dutto y Gastañaga, 2019) y se basa en tres pilares para generar su actividad económica: impacto social, impacto medioambiental e impacto económico.

El presente artículo tiene como objetivo analizar el desarrollo de las empresas o sociedades BIC en Perú, luego de la publicación de la Ley 31072 «Ley de la Sociedad de Beneficio e Interés Colectivo» (El Peruano, 2020) y su reglamento, publicado por Decreto Supremo N ${ }^{\circ} 004-2021$ PRODUCE (Ley $\mathrm{N}^{\circ} 31072,2021$ ), bajo una óptica de legislación y administración comparada con países de Iberoamérica y América del Norte: España, Colombia, Ecuador, Argentina, Chile, México, Uruguay, El Salvador, Estados Unidos de América y Canadá. Para ello, se recurre a una revisión bibliográfica en repositorios gubernamentales y académicos.

\section{Evolución del desarrollo sostenible en las empresas}

La preocupación por asegurar la integridad de las futuras generaciones tiene un origen remoto fundado en principios morales y filosóficos (Canessa y García, 2021). No obstante, hasta la década de 1950, las actividades actualmente conocidas como de responsabilidad social eran interpretadas como actos caritativos de una persona individual, incluso cuando las actividades eran realizadas en un contexto empresarial (Aldeanueva-Fernández, 2014). Solo para el año de 1953, gracias al aporte de Bowen, se vislumbra una aproximación con respecto a los objetivos a largo plazo e intereses de la sociedad (Posada, 2013) que con el correr del tiempo desembocarían en las empresas societarias BIC.

De acuerdo con Caravedo (2017), la evolución del propósito en las empresas podría dividirse en tres momentos. Sin embargo, se puede notar una cuarta categorización dentro de su sustentación, donde se diferencia entre una empresa que realiza actividades en aras de la sociedad y una empresa certificada que realiza esas actividades. En la tabla 1 se muestran distintos enfoques del desarrollo sostenible en las empresas.

Se percibe que el impacto del empresariado dentro de la sociedad puede ser un impacto único, fundado netamente en tópicos económicos; un impacto doble, donde además de la rentabilidad se considera el ámbito social o 


\section{Tabla 1}

\section{Esquema de evolución del desarrollo sostenible en las empresas}

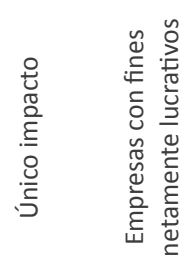

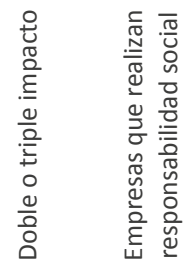

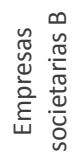

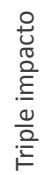

Estructura jurídica: empresa individual o sociedades

Fines de utilidad: (re)inversión y/o dividendos

Distribución presupuestaria: desarrollo de sus actividade

Presentación de informes de RSE: no

Obligación de realización de actividades de RSE: no

Estructura jurídica: empresa individual o sociedades

Fines de utilidad: (re)inversión, dividendos y/o actividades sociales

Distribución presupuestaria: desarrollo de sus actividade incluyendo responsabilidad social Presentación de informes de RSE: interna de acuedo a su gestión Obligación de realización de actividades de RSE: no

\section{Estructura jurídica: sociedades}

Fines de utilidad: (re)inversión, dividendos y/o actividades sociales

Distribución presupuestaria: desarrollo de sus actividade incluyendo responsabilidad social Presentación de informes de RSE: interna y externa (a la organización certificadora) de acuedo a su gestión

Obligación de realización de actividades de RSE: sí

Estructura jurídica: sociedades BIC

Fines de utilidad: (re)inversión, dividendos y/o actividades sociales

Distribución presupuestaria: desarrollo de sus actividade incluyendo responsabilidad social Presentación de informes de RSE: interna y externa (al estado) de acuedo a su gestión Obligación de realización de actividades de RSE: sí

Nota. Natural Gas Supervision Division DGH-Minem. Annual Book of Hydrocarbon Resources, 2018. 
ambiental; o, un impacto triple, que incluye los aspectos de rentabilidad, social y ambiental. (Bajo, González y Fernández, 2013)

Asimismo, se observa que las empresas B implican el reconocimiento por entes certificadores (Salmeron, 2019), con la presentación de informes y documentación a estas entidades para evaluar su capacidad de aportación a un entorno más amigable con el ambiente, mientras que las sociedades BIC son una forma jurídica de empresa, que tienen el reconocimiento e identidad de ser organizaciones con un interés económico, social y ambiental, en la realización de sus actividades (Mujica, 2016).

\section{Escenario internacional}

A continuación, se presenta un análisis de la legislación de diversos países (que muestran un mayor desarrollo del tema que Perú) sobre el desarrollo sostenible en las empresas:

Estados Unidos de América. En este país surge la concepción más próxima a las sociedades BIC, reconocidas a través de «B-Corp Certificate» (Herrera y Diaz, 2008). Sin embargo, las empresas societarias B no tienen el mismo marco jurídico que una empresa societaria BIC (Caravedo, 2017). Actualmente, dentro de los Estados Unidos de América existe un marco regulatorio para las empresas BIC, bajo el nombre de «Public Benefit Corporations», algunas veces llamadas «Benefit Corporations», «B-Corporations», «B-Corp»; pese a la proximidad lingüística, una empresa societaria reconocida por «B-Corp Certificate» no necesariamente es una «Public Benefit Corporations» (Hayes, 2019). No obstante, al año 2020, esta forma societaria no estaba reconocida en todos los Estados, sino solamente en 35 (Bernier et al., 2020). Sin embargo, cabe destacar que existe un deseo de reconocimiento por este marco, pues para el año 2021 está amparado en 37 Estados y con propuestas legislativas en cuatro más (Benefit Corporation, 2021). Las obligaciones y beneficios pueden diferir en algunos aspectos, pero en su mayoría siguen los mismos lineamientos: inclusión de algún beneficio público en su estatuto, dirección colaborativa, transparencia contable (Chen, 2021). Cabe notar que no todos los Estados permiten el paso de una «C-Corporation» (tradicional) a una «Public Benefit Corporation» (Legal Information Institute, 2020).

España. En España se comenta que el comportamiento responsable de las empresas contribuye al cumplimiento de los ODS (Remacha, 2017) y, consecuentemente, al beneficio colectivo. Sin embargo, no existe un marco jurídico que ampare a las empresas de triple impacto (Boletín Oficial del Estado, 2010). A pesar de haberse presentado en dos oportunidades para la inclusión de las empresas societarias BIC en la Ley de Sociedades Comerciales, esta figura no ha sido aprobada por el Senado español (Pizzaferri, J., Diaz, M., Vázquez, M., Yunes, M., Gómez, N. y Tesoro R. (2021). No obstante, con respecto, a las empresas societarias B, Pablo Sánchez, director B Lab Spain (ONG encargada de la certificación de empresas B) sostiene que España está demostrando que se puede construir una nueva realidad donde el centro sean las personas y el planeta desde el ente privado, y se hace referencia a un crecimiento de 74 a 100 empresas del 2020 al 2021 (Corresponsables, 2021). Por otro lado, debe mencionarse que, dentro del marco jurisdiccional (Ley 11/2018, 2018), se cuenta con las denominadas Entidades de Interés Público (EIP), que, si bien no comprenden a las empresas societarias BIC, mantienen ciertas similitudes, pues con ellas se fomenta 
la divulgación no financiera (INGERTEC, 2019). Entre la información que debería contar el informe anual de gobierno corporativo, obligatorio para este tipo de organizaciones, se puede encontrar políticas de diversidad de competencias y puntos de vista, consideraciones medioambientales y sociales, derechos humanos, así como a la lucha contra la corrupción y el soborno (Ley 11/2018, 2018). No obstante, debe tomarse en cuenta que, de acuerdo con Real Decreto 877/2015, de desarrollo de la Ley 26/2013, para ser considerado como una EIP, se exige también que se trate de una entidad de crédito, aseguradora, emisora de valores, prestadora servicios de inversión (a más de 5,000 personas), gestora de fondos de pensión, entidad de pago, o alguna otra cuyo importe neto de la cifra de negocios y plantilla media durante dos ejercicios consecutivos, a la fecha de cierre de cada uno de ellos, sea superior a 2,000 millones de euros y a 4,000 empleados, o pertenecer a un grupo de sociedades donde la dominante contemple alguna de las categorizaciones anteriores(Real Decreto 877/2015, 2015). Ello conllevaría a que la empresa cumpla funciones bancario-contables y/o sea de tamaño de mediano a grande y cotizable en bolsa, imposibilitando a otras a enmarcarse como EIP. Sin embargo, debe decirse que este marco jurisdiccional cumple con su objetivo debido a que procura brindar veracidad y transparencia a los stakeholders en aras de evitar crisis económica (Maza, 2019)

Colombia. En Colombia, se encuentra la primera aproximación de Latinoamérica para el establecimiento de un marco jurídico que identifique y respalde a las organizaciones de triple impacto, a través de la propuesta de ley del Centro Democrático, en el año 2016 (Lancheros y Tolé, 2021). Su creación no se daría sino hasta junio de 2018, bajo la Ley 1901 de «Sociedades Comerciales de Beneficio e Interés Colectivo (BIC)» ( Ley 1901, 2018), con un reglamento que entraría en vigencia por Decreto 2046 en 2019 (Moreno, 2020). Se reconocieron tres objetivos fundamentales: un propósito social y ambiental, la realización de ese propósito y la transparencia de reportes de impacto empresarial (Calixto, 2019). En el desarrollo de la ley, se puede apreciar una aproximación a la satisfacción bajo los estándares de Maslow, comenzando por el cumplimiento de las necesidades fisiológicas hasta la realización personal y sentido de trascendencia (Delgado y Romero, 2020), cumpliendo con las vertientes del salario emocional (RubioRodríguez, G. y Varón Romero, 2018). Por otro lado, y en un enfoque más empresarial, se reconoce que la denominación como empresa BIC trae consigo acceso preferente a líneas de crédito del Estado, deducción y excepción de impuestos (Presidencia de la República de Colombia, 2019), acceso preferente a convocatorias, diferenciación en plataforma «Compra lo nuestro» (Ministerio de Comercio, Industria y Turismo, 2021), optimización de las relaciones públicas (Legis Comunidad Contable, 2019) y mayor atracción a la inversión (Giraldo Angel Asociados, 2019). Cabe notar que la denominación BIC es una condición, más no un tipo societario nuevo: «Su creación no implica un cambio de tipo societario, tampoco corresponde a un tipo societario nuevo» (Superintendencia de Sociedades, 2019).

Argentina. Con normativa aún no vigente respecto a sociedades BIC (Tuchin, 2021), el empresariado argentino ha sabido cómo desarrollar sus actividades de la mano de las tendencias de triple impacto, a través del reconocimiento de 131 empresas como empresas B certificadas por Sistema B (Sistema, 2021). Desde 2016, se han presentado diferentes proyectos de ley para 
la concertación del marco regulatorio, nueva forma de organización empresarial: las sociedades de interés y beneficio colectivo (BIC), y modificatoria de la Ley 19.550 «Ley General de Sociedades». En el primer caso, no se llegó a discutir, y en el segundo, se consideró que la propuesta de solo nueve artículos era incompleta y que no podría ser concretada (Cracogna, 2019). Por otro lado, se aprecia la posición de juristas que sostienen la no necesidad de generar un nuevo marco jurídico, debido a que, dentro de la Ley 19.550 ( Ley general de sociedades $\left.\mathrm{N}^{\mathrm{o}} 19.550,2014\right)$, la creación de este tipo de organizaciones podría estar enmarcada en otra figura y los tres valores de las sociedades BIC podrían ser incluidos dentro de cualquiera de las otras, además de contar con la posibilidad de certificación B (Basualdo, 2019). Siendo así, es de notar que no se contaría con los beneficios crediticios e impositivos que se mencionan en los proyectos de ley (Pereyra, 2019).

Ecuador. En Ecuador, se muestra a las sociedades BIC como organizaciones que presentan un impacto concreto en la sociedad y en el ambiente (Griffin, 2020), que es corroborado a través de un informe de validez internacional (por ejemplo, Global Reporting Initiative) y con la difusión plena de los estados contables, a diferencia de la legislación de otros Estados (Álvarez et al., 2021). De esta forma, desde el 2020, las sociedades BIC están amparadas por la Ley Orgánica de Emprendimiento e Innovación (Sistema B, 2020). También se percibe que una Sociedad BIC es una condición y que no conllevaría el cambio de figura jurídica tipificada en la Ley de Compañías (Ley orgánica de emprendimiento e innovación, 2020). Cabe mencionar que, dentro de la presente, no se percibe ningún tipo de beneficio estatal para las empresas que opten por tornarse una sociedad BIC; se trata de un punto que se puede fortalecer en aras de generar una mayor acogida (Lecaro, 2021).

Canadá. En Canadá, además del recurso a empresas certificadoras de organizaciones $\mathrm{B}$, se tiene respaldo legal a través de las Compañías de Contribución Comunitaria o «Community Contribution Companies», modelo organizacional creado en British Columbia en 2013, que brindan identidad jurídica a los emprendedores sociales (Horel y McKague, 2018). Esto se encuentra en el documento «Business Corporations Act», Capítulo 57, y parte 2.2 (Business Corporations Act, 2021). De esta manera, una región geográfica del país tendría el amparo legal para promover el desarrollo de una sociedad de beneficio e interés colectivo. Sin embargo, el resto del país no tendría tal figura, de manera que el beneficio a los grupos de interés de la sociedad sería opcional, salvo se siga la ruta de una certificación B que obliga a construir valor para la comunidad (Sustainable Prosperity, 2012). Por otra parte, se tiene una crítica a las «Benefit Corporations», dado que para el modelo de gobernanza corporativa canadiense sería redundante integrar esta figura organizacional. Esto se debe a que los directores deben actuar basados en los mejores intereses de la compañía, tomando en cuenta, además, la declaración del Ministerio de Innovación, Ciencia y Desarrollo Económico, de Canadá, de que una empresa social persigue metas sociales, culturales y ambientales, a través de la venta de bienes y/o servicios. Se destaca la utilización de la mayoría de las utilidades netas para conseguir un objetivo social, con distribución limitada para accionistas y dueños (Liao, 2017).

México. En México, se presenta una situación en la que no se tiene un marco legal fuerte para impulsar el desarrollo de empresas sociales; no existe una referencia clara a este figura en su 
Ley General de Sociedades Mercantiles (Ley General de Sociedades Mercantiles, 2009). Además, la producción académica respecto a organizaciones de beneficio e interés colectivo en el país es limitada. Sin embargo, existen asociaciones civiles que promueven procesos de transformación y sustentabilidad para la reducción de pobreza en México y para vincular empresas líderes y empresas sociales (de modo que se construya valor económico, social y ambiental), como es el caso de la Fundación del Empresariado en México (Cáceres, Sosa y Pereyra, 2018). Lo más cercano a una ley que tenga relación con las empresas de beneficio e interés colectivo es la «Ley de la Economía Social y Solidaria», publicado en el 2012, que promueve el desarrollo de la economía social en México (Ley de la Economía Social y Solidaria, 2019). No obstante, no enfatiza algún modelo jurídico que las empresas puedan adoptar respecto al beneficio de triple impacto, sino que se enfoca en la equidad social y productividad (Guerra, 2012).

El Salvador. En El Salvador, se cuenta con una sede de Sistema B (Sistema B, 2020), una empresa que certifica a empresas de triple impacto en América Latina y el Caribe. Si bien no se encontró información académica sobre el desarrollo de iniciativas de sociedades de beneficio e interés colectivo, se observa que la responsabilidad social empresarial está mejorando en El Salvador, con la presencia de códigos de ética y programas de reciclaje (Fundación Salvadoreña para el Desarrollo Económico y Social, 2014). No obstante, la regulación legal sigue siendo un faltante para promover una participación más activa de las organizaciones en el triple impacto; por el momento, sólo se tiene la Ley de Cooperativas y el Código de Comercio para el desarrollo de la Economía Social Solidaria en El Salvador
(Alianza Cooperativa Internacional para las Américas, 2007), que no satisface plenamente las condiciones de una sociedad de beneficio e interés colectivo.

Uruguay. En Uruguay, se cuenta con la Ley $\mathrm{N}^{\circ}$ 19969 sancionada por el Senado uruguayo el 14 de julio de 2021, que regula la actividad de las empresas BIC o de triple impacto, teniendo en su denominación la expresión de «beneficio e interés colectivo» o «BIC» (Ley $\mathrm{N}^{\circ} 19969$, 2021). Los administradores o fiduciarios deben desarrollar un reporte anual, en el que se acredite las actividades que respaldan su propósito social y ambiental en la consideración de su giro de negocio (PwC, 2021). Si es que no se cumplen las condiciones, se procede a la descalificación producida de oficio por la autoridad competente, al verificarse el incumplimiento de responsabilidades de sociedad BIC (Centro de Información Oficial, 2021). Si bien la ley no ofrece algún beneficio directo inmediato, las empresas pueden aprovechar su estatus para gestionar relaciones con los clientes, a través de la responsabilidad social empresarial (Ferre, et al., 2011).

Chile. En Chile, aún no se cuenta con una normativa legal para tratar a las empresas BIC; sin embargo, se cuenta con un gran número de organizaciones B certificadas, liderando en Latinoamérica, para el 2017, con 99 empresas B (Biblioteca del Congreso Nacional de Chile, 2017). La iniciativa del poder legislativo se presentó en el Congreso de la República de Chile para su evaluación el 6 de octubre de 2015, y también el 13 de junio de 2017 (El Mercurio, 2020). Chile tiene una historia de Responsabilidad Social Empresarial construida de manera sostenida, llegando a ser un pionero en Sudamérica (Posada, 2013), por lo que una regulación que ampare a las socie- 
dades de beneficio e interés colectivo se puede desarrollar sobre la base de sus experiencias y conocimiento. Finalmente, la Ley 18046 «Ley sobre Sociedades Anónimas», indica que una sociedad anónima será siempre mercantil, incluso si tiene negocios de carácter civil (Ley 18046, 2021), lo que sugiere la necesidad de reforzar el marco regulatorio para la promoción de organizaciones de triple impacto.

\section{Tabla 2}

\section{Características comparadas}

\begin{tabular}{ccc}
\hline País & Beneficios por la condición \\
de empresa BIC & Desarrollo en general
\end{tabular}

Estados Unidos de América

\section{Se ampara el modelo} societario en cuestión en 37 Estados bajo el nombre de «Public Benefit Company».
Sí, varía de acuerdo al Estado, pero, tienden a ser accesos preferentes y deducciones impositivas.
El país es uno de los líderes en empresas de triple impacto, con un gran nivel de capacidad de inversión en innovación sostenible.
No se presenta alguna ley en vigor que regule la modalidad de empresas BIC.

España Hubo propuestas legislativas y cuenta con un marco jurisdiccional relativamente similar: Entidades de Interés Público (EIP).

Ley 1901, del 18 de junio de

Colombia 2018, que otorga la existencia jurídica de las empresas $B$.

No se presenta alguna ley en Argentina vigor que regule la modalidad de empresas BIC. Hubo propuestas legislativas.
Sí, tarifas preferenciales para servicios de propiedad intelectual, deducción impositiva, líneas de crédito, acceso preferente a convocatorias, diferenciación, entre otros.

No corresponde.
El gobierno español promueve la sostenibilidad en las organizaciones, con un apoyo político para el desarrollo de empresas BIC $o$ afines.
Las empresas que se alinean con los ODS tienen el reconocimiento de un ministerio del gobierno colombiano que impulsa su construcción organizacional.
La iniciativa privada promueve el desarrollo de organizaciones B para la gestión de actividades empresariales. 
La Asamblea Nacional de Ecuador aprobó,

Ecuador unánimemente, la Ley de Emprendimiento e Innovación que reconoce a las sociedades BIC en 2020.

Se ampara el modelo societario en cuestión en

Canadá

México

No se presenta alguna ley en vigor que regule la modalidad de empresas BIC.

No se presenta alguna ley en

El Salvador vigor que regule la modalidad de empresas BIC.

Ley de Sociedades de Beneficio e Interés Colectivo, sancionada el 14 de julio de 2021.

No se presenta alguna ley en

Chile vigor que regule la modalidad de empresas BIC. Hubo propuestas legislativas.
En evaluación.

Las organizaciones BIC se desarrollan por el desenvolvimiento de la iniciativa privada, para alcanzar un triple impacto en el país, con el respaldo de la organización certificadora Sistema B.

\section{Canadá tiene un gran} desarrollo numérico de En evaluación. empresas tipo B; ocupa uno de los primeros lugares en el mundo.

\section{Se presenta un impulso} desde el área privada para generar modelos sostenibles de negocio.

No se cuenta con un gran nivel de desarrollo de empresas B; sin embargo, se tiene la iniciativa privada, con una sede de la empresa certificadora Sistema B, para un futuro progreso en este campo.

Las organizaciones deben realizar los reportes necesarios para confirmar su sociedad de beneficio e interés colectivo.

Chile es uno de los países con mayor número de empresas B certificadas, con el quinto puesto a nivel mundial en términos cuantitativos.

Nota. Elaboración propia. 


\section{Escenario doméstico}

Las necesidades de innovación y búsqueda de desarrollo sostenible han dado espacio a la concepción de sociedades BIC; no obstante, pese a la notoria tendencia internacional que reconoce la relevancia de los principios del desarrollo sostenible y su estrecha relación con el empresariado, el Perú no contaba hasta hace un par de años con una regulación al respecto. En Perú, la incipiente regulación ocasionaba que muchas personas jurídicas constituidas dentro de cualquiera de los siete tipos societarios regulados en la Ley General de Sociedades (LGS), prefiriesen modificar la estructura y procesos de su organización externa e internamente con la finalidad de convertirse en una empresa B Certificada, como camino que permitía a las personas jurídicas llevar a cabo el desarrollo de un propósito de beneficio social y medioambiental determinado (Mujica, 2016).

Por otro lado, Elizabeth Ü señala que la persona jurídica encargada de la certificación, B Lab, resulta ser de gran ayuda para la creación de normativa relacionada con las sociedades $\mathrm{BIC}$ en los países en donde aún no existe una legislación (Ü, 2013). Tanto las sociedades BIC como las empresas B trabajan con la finalidad de lograr un impacto positivo en la sociedad y el medioambiente.

Adicionalmente, podemos observar el impacto que tuvieron las empresas B en América estos últimos 10 años, donde Latinoamérica tuvo el $13.3 \%$ de las empresas B Certificadas mundialmente (Caravedo, 2016). Asimismo, es importante señalar que los países de Latinoamérica que tienen más empresas B son aquellos que han logrado regular en sus ordenamientos jurídicos las sociedades de beneficios e interés colectivo.
Según Caravedo, Perú tuvo una cantidad menor de empresas B en comparación con otros países, sin embargo, el número de empresas que han consultado la evaluación a E Lab en el Perú ha ido creciendo en estos últimos diez años en un $22.25 \%$ (incremento total) respecto del año 2011, lo que sugiere el interés de las empresas peruanas por cambiar la empresa tradicional a una empresa con propósito, que genere un impacto positivo en la sociedad (Caravedo, 2016). En el año 2019, Perú registraba 28 empresas B en diferentes industrias, todas buscando alcanzar sus fines económicos, sociales y ambientales con un propósito de beneficio (Vilchez, 2019).

Es así que, hace menos de dos años el ordenamiento jurídico peruano aún no regulaba la figura de sociedades BIC; el punto de quiebre fue el proyecto de ley $\mathrm{N}^{\circ} 2533 / 2017-\mathrm{CR}$ presentado el de marzo del 2018, que tuvo como resultado la publicación de la Ley $\mathrm{N}^{\circ}$ 31072, Ley de la Sociedad de Beneficio e Interés Colectivo, el 24 noviembre de 2020 (Ley $\left.\mathrm{N}^{\circ} 31072,2020\right)$, entrando en vigencia su Reglamento el 23 de febrero de 2021, aprobado por el Decreto Supremo N004-2021PRODUCE (El Peruano, 2021).

En esa línea, las personas jurídicas societarias existentes o por constituirse que decidan acogerse a la ley de Sociedades BIC deberán cumplir los requerimientos señalados en la tabla 3.

La ley de sociedades BIC no otorga ningún beneficio tributario a las personas jurídicas que opten por esta nueva figura; sin embargo, se espera que estos beneficios se originen a partir del Estado, como se podría dar el caso en las contrataciones donde las municipalidades valoren estas empresas de interés colectivo (De La Torre, 2021). 


\section{Tabla 3}

Requerimientos para convertirse en Sociedades BIC según la Ley $N^{\circ} 31072$

Denominación de Sociedades BIC

Manifestación expresa

Propósito de beneficio

Modificación del estatuto de la persona jurídica existente
El artículo 4 de la ley señala que cada persona jurídica societaria deberá agregar a su denominación el término «de beneficio e interés colectivo» o, en su defecto, la sigla «BIC».

El artículo 5 inciso 1 de la ley establece que las personas jurídicas que decidan acogerse a la presente ley deberán señalarlo en su pacto social y estatuto. Aunado a ello, deberán inscribirlo en el registro de personas jurídicas del domicilio correspondiente.

El artículo 5 inciso 2 en concordancia con el artículo 6, señala que el estatuto de sociedad BIC deberá constar una descripción detallada del propósito de beneficio, ello debe estar después del artículo que regule su objeto social.

El artículo 5 inciso 3 señala que, para la modificación del estatuto, las personas jurídicas requerirán:

1. En primera convocatoria, cuando menos, la concurrencia de dos tercios de las acciones suscritas con derecho a voto y, en segunda convocatoria, se necesitará la concurrencia de por lo menos tres quintas partes de las acciones suscritas con derecho a voto, o la mayoría calificada según lo requiera cada tipo societario o el estatuto. 2. En caso la sociedad decida cambiar el propósito de beneficio o su condición de sociedad BIC, ello requerirá la misma formalidad establecida en su constitución.

3. Asimismo, el socio o accionista que manifiestamente se encuentre en desacuerdo con el cambio en el estatuto de la sociedad podrá ejercer el derecho de separación de acuerdo con las disposiciones de la Ley General de Sociedades, Ley 26887.

Nota. Adaptado de la Ley N 31072 «Ley de la Sociedad de Beneficio e Interés Colectivo».

Finalmente, en Perú se comenta que la ley BIC otorga identidad jurídica a las empresas de triple impacto (Caillaux, 2020) y que las sociedades BIC pueden cumplir un rol estratégico para alcanzar los Objetivos de Desarrollo Sostenible (ODS) de la ONU. Mediante esta figura, se podría lograr un régimen económico más resiliente, que incluya estándares de calidad ambiental e inclusión social, así como fortalecer el sistema de salud (Caillaux y Ochoa, 2021).

\section{Conclusiones}

Las empresas BIC, como empresas que procuran un beneficio integral de la sociedad, son la conceptualización más reciente de responsabilidad social empresarial, y cuentan con un marco 
jurídico que las ampara en 5 de los 10 países considerados en este estudio: Estados Unidos de América, Colombia, Ecuador, Canadá y Uruguay; asimismo, se han presentado propuestas en España, Argentina y Chile, y están por elaborarse en México y El Salvador.

Existe una gran diferencia entre las empresas BIC y su antecesor histórico en la evolución de la responsabilidad social empresarial, las empresas B. Las empresas B son reconocidas por organizaciones certificadoras y su respaldo está fundado en una cosmovisión global o regional, como B-Corp o Sistema B. Por su parte, las empresas BIC son reconocidas por los propios gobiernos nacionales. Entonces, es posible que una empresa BIC sea a su vez una empresa B, pero, no son términos que puedan emplearse indistintamente, a pesar de tener obligaciones muy similares para mantener la denominación de B y/o BIC: realización de actividades en pro de la sociedad y/o ambiente, y presentación de informes de impacto y económicos.

Cabe notar que en algunos de los países estudiados (Estados Unidos de América y Colombia) las sociedades BIC pueden percibir diferentes beneficios, como acceso preferente a líneas de crédito del Estado, deducción y exención de impuestos, más allá de los beneficios en relaciones públicas y atracción de inversiones. Sin embargo, en otros países (Ecuador, Uruguay y Canadá) aún los incentivos están en proceso de evaluación.

En el escenario peruano, se percibe que la Ley $\mathrm{N}^{\circ} 31072$, Ley de la Sociedad de Beneficio e Interés Colectivo del 24 noviembre de 2020, y su reglamento correspondiente, Decreto Supremo N $^{\circ}$ 004-2021-PRODUCE, amparan a las empresas societarias BIC; su promulgación es fruto del desarrollo internacional comparado y la incidencia de las empresas certificadas $\mathrm{B}$ en el ámbito nacional. Sin embargo, existe un problema de incentivos para la transición y continuidad de este modelo. No se percibe más beneficio que el reconocimiento, lo cual podría ser insuficiente tomando en cuenta la gestión adicional demandada por los requerimientos estatales. Este es un punto que ralentiza la adopción de la forma jurídica. 
Álvarez Gavilánez, J. E., Montes De Oca, J. E., Murillo Párraga, D. y González Ortega, R. (2021). Dimensiones para las sociedades de bienestar e interés colectivo del Ecuador. Revista Universidad y Sociedad, 13(S1), 389-397.

Bajo, A., González, M. y Fernández, J. L. (2013). Responsabilidad social y empresa sostenible. AdComunica, 223-243. https://doi.org/10.6035/2174-0992.2013.5.14

Basualdo, M. (4-6 de septiembre de 2019). ¿Es necesaria la regulación de las sociedades de beneficios e interés colectivo (BIC)? Congreso Argentino de Derecho Societario y X Congreso Iberoamericano de Derecho Societario y de la Empresa, Rosario, Argentina. https://goo.su/9puP

Benefit Corporation. (2021). State by state status of legislation. https://goo.su/9yQJ

Bernier, P., Lebovitz, M., Ikharo, T. y Hurst, S. (2020). A primer on public benefit-focused corporate models in California and beyond. Mayer Brown. https://goo.su/9rng

Biblioteca del Congreso Nacional de Chile. (2017). Empresas B: Antecedentes generales y regulación comparada en Colombia, Argentina y Estados Unidos de América. https://goo.su/RuW

Business Corporations Act. (2021). 24 de noviembre de 2021. Canadá. https://www.bclaws.gov.bc.ca/civix/ document/id/complete/statreg/02057_03a

Cáceres, A., Sosa, M. y Pereyra, A. (2018). La contribución de una empresa tipo B hacia la sustentabilidad en Mérida, Yucatán. En Agenda Pública para el Desarrollo Regional, la Metropolización y la Sostenibilidad. (2). https://goo.su/9JEv

Caillaux, J.(2020). Mejores empresas para el Perú. [Diapositivas de Power Point]. https://goo.su/akHw

Caillaux, J., Ochoa, C. (2021). Empresas con propósito social y ambiental. En L. Raez (Ed.), Proyecto Perú Debate 2021: propuestas hacia un mejor gobierno. https://goo.su/9GS6

Calixto, D. (5 de diciembre de 2019). Nueva reglamentación de las Sociedades de Beneficio e Interés Colectivo -BIC(Decreto 2046 de 2019). Guerrero Ruiz Asociados. https://goo.su/9HRs

Canessa, G. y García, E. (2021). El ABC de la responsabilidad social empresarial en el Perú y en el mundo. Biblioteca Nacional del Perú: Lima. https://goo.su/9YWf

Caravedo, B. (2016). La energía social en las empresas B. Lima: Universidad del Pacífico.

Caravedo, B. (2017). La energía social de las empresas B. Revista de Ciencias de la Gestión, 1(2), 153-158.

Chen, J. (2021). What is a California benefit corporation?. The Law Office of Jeremy Chen. https://goo.su/9WGg

Corresponsables. (11 de agosto de 2021). ODS17. La incorporación de empresas al movimiento B Corp en España crece por encima de la media mundial. https://goo.su/9wuB

Cracogna, D. (4-6 de septiembre de 2019). El proyecto de ley de sociedades BIC. Congreso Argentino de Derecho Societario y X Congreso Iberoamericano de Derecho Societario y de la Empresa, Rosario, Argentina. https:// goo.su/SaK 
De La Torre, A. (20 de enero de 2021). Los detalles de la nueva ley de sociedades BIC en el Perú. LexLatin. https:// goo.su/9Nt4

Delgado, E. C. y Romero, N. (2020). Fines de la creación y desarrollo de las sociedades de beneficio e interés colectivo (BIC) en Colombia. [Tesis para optar el grado de abogado] Universidad de Los Andes. https://goo. $\mathrm{su} / 9 \mathrm{Hvh}$

El Peruano. (2021). Decreto Supremo que aprueba el Reglamento de la Ley N 31072, Ley de la Sociedad de Beneficio e Interés Colectivo (SOCIEDAD BIC). 22 de febrero de 2021. Perú. https://busquedas.elperuano. pe/normaslegales/decreto-supremo-que-aprueba-el-reglamento-de-la-ley-n-31072-decreto-supremo-n004-2021-produce-1929774-4/

El Mercurio. (2020). Movimiento legislativo de sociedades de beneficio e interés colectivo (B.I.C.) en América Latina: análisis, desafíos y propuestas para su avance. http://static.elmercurio.com/Documentos/ Legal/2020/08/21/20200821112735.pdf

Emas, R. (2015). Brief for GSDR 2015. The concept of sustainable development: definition and defining principles. Florida International University. https://goo.su/adQA

Ferre, Z., Melgar, N., Pastori, H., Piani, G. y Rossi, M. (2011). La responsabilidad social empresarial en Uruguay: una visión comparada desde la ciudadanía y desde las empresas. Semestre económico, 14(30), 105-120. https://goo.su/9Gr5

Fusades. (2014). Responsabilidad social empresarial en El Salvador: prácticas y estrategias para prevenir el crimen y la violencia. https://goo.su/Gjg

Giraldo Angel Asociados. (26 de noviembre de 2019). Decreto 2046 de 2019 por medio del cual se impulsan las empresas BIC en Colombia. https://goo.su/9ZRS

Griffin, A. (2 de marzo de 2020). Ley de emprendimiento e innovación: Impulso al emprendimiento y regulación de las SAS. Pérez, Bustamante y Ponce. https://goo.su/a6Sd

Guerra, P. (2012). Las legislaciones sobre economía social y solidaria en América Latina Entre la autogestión y la visión sectorial. Revista de la Facultad de Derecho. 33. 73-94. https://goo.su/sgD

Hayes. J. (13 de noviembre de 2019). What's the difference between a public benefit corporation and a B corp certification?. Hutchison. https://goo.su/9fxa

Herrera, A. y Díaz, M. (2008). Dimensionalidad de la responsabilidad social empresarial percibida y sus efectos sobre la imagen y la reputación: una aproximación desde el modelo de Carroll. Estudios gerenciales, 24(108), 37-59.

Horel, B. y McKague, K. (2018). Community contribution companies and access to social finance. Journal of sustainable development. 11(5). doi:10.5539/jsd.v11n5p126

INGERTEC. (2019). ¿Sabes si tu empresa es una entidad de interés público?. https://goo.su/9V40

Lancheros, P., y Tole, J. (2021). Empresas BIC en Colombia: una luz en la implementación de los Estándares Interamericanos en DDHH y Empresas. Homa pública - Revista internacional de derechos humanos y empresas, 5(1). https://goo.su/b9My 
Lecaro, J. (2021). Los mecanismos legales de la administración pública en materia de incentivos económicos: estudio especial a las sociedades BIC en Ecuador. [Tesis para optar el grado de abogado. Universidad Católica de Santiago de Guayaquil]. https://goo.su/tro

Legal Information Institute. (noviembre de 2020). Public benefit corporation. https://goo.su/9HN4

Legis Comunidad Contable. (22 de noviembre de 2019). Precisiones del Decreto 2046 de 2019, que busca impulsar las empresas BIC en Colombia. https://goo.su/aU2I

Ley $N^{\circ}$ 31072. (2020). Ley de la sociedad de beneficio e interés colectivo. (Sociedad BIC). 2 de noviembre de 2020. https://goo.su/9tE9

Ley N 19969. (2021). Creación de las sociedades de beneficio e interés colectivo (BIC). 23 de julio de 2021. Uruguay. https://goo.su/9VKL

Ley 18046. (2021). Ley sobre sociedades anónimas. (13 de abril de 2021). https://goo.su/9zQ2

Ley 1901 (2018). Por medio de la cual se crean y desarrollan las Sociedades Comerciales de Beneficio e Interés Colectivo (BIC). 18 de junio de 2018. Colombia https://www.funcionpublica.gov.co/eva/gestornormativo/ norma.php?i=86982

Ley 11/2018. (2018). Por la que se modifica el Código de Comercio, el texto refundido de la Ley de Sociedades de Capital aprobado por el Real Decreto Legislativo 1/2010, de 2 de julio, y la Ley 22/2015, de 20 de julio, de Auditoría de Cuentas, en materia de información no financiera y diversidad. 28 de diciembre de 2018. https://goo.su/9iaT

Ley de la Economía Social y Solidaria, reglamentaria del párrafo octavo del artículo 25 de la Constitución Política de los Estados Unidos Mexicanos, en lo referente al sector social de la economía. (2019). 12 de abril de 2019. México https://goo.su/Xoz

Ley general de sociedades № 19.550, T.O. 1984. (2014). Ley de sociedades comerciales. 19 de diciembre de 2014. Argentina. https://goo.su/9yE2

Ley general de sociedades mercantiles. (2009). 30 de abril de 2009. México. https://goo.su/a2WI

Ley orgánica de emprendimiento e innovación. (2020). 21 de febrero de 2020. Suplemento del Registro Oficial No. 151. Ecuador. https://goo.su/YXo

Liao, C. (2017). A critical Canadian perspective on the benefit corporation. Faculty publications at Allard research commons, 4O(2). https://goo.su/9WXD

Maza, D. (2019). La auditoría de cuentas en entidades de interés público: análisis de las empresas cotizadas en la Bolsa de Madrid. [Tesis para optar el grado en administración y dirección de empresas. Universidad de Cantabria]. https://goo.su/9MnL

Ministerio de Comercio, Industria y Turismo. (18 de marzo de 2021). ¿Cuáles son los beneficios de ser una Sociedad BIC?. https://goo.su/9S2k 
Moreno, J. (2020). Ley de sociedades comerciales de beneficio e interés colectivo: ¿un avance para un régimen jurídico de responsabilidad social empresarial en Colombia?. [Tesis para optar el grado de abogado. Universidad EAFIT]. https://goo.su/9wTW

Mujica, J. D. (2016). Sociedades de beneficio e interés colectivo: un aporte societario al bienestar social y medioambiental. [Tesis para optar el grado de abogado. Universidad de Lima]. https://goo.su/aaau

Parlamento del Uruguay. (2021). Sociedades beneficio e interés colectivo (BIC). https://parlamento.gub.uy/ documentosyleyes/ficha-asunto/147986

Pereyra, A. (4-6 de septiembre de 2019). Sociedades de beneficio e interés colectivo. Sociedades BIC o de triple impacto. El compromiso de nuestro país al pacto mundial de Naciones Unidas. Congreso Argentino de Derecho Societario y $X$ Congreso Iberoamericano de Derecho Societario y de la Empresa, Rosario, Argentina. https://goo.su/9TnG

Pizzaferri, J., Diaz, M., Vázquez, M., Yunes, M., Gómez, N. y Tesoro R. (2021). Algunas consideraciones sobre el proyecto de Ley de Sociedades de Beneficio e Interés Colectivo. Comisión de estudios sobre sustentabilidad económica, social y medioambiental. https://goo.su/YkO

Posada, A. (2013). Chile, pionero en temas de responsabilidad social en Sur América. Cultura, Educación y Sociedad, 4(1), 41-53. https://goo.su/9RqP

Presidencia de la República de Colombia (12 de noviembre de 2019). Abecé del Decreto 2046 de 2019 que busca impulsar las empresas BIC en Colombia. https://goo.su/PPG

PWC. (2021). Novedades Impuestos y Legal. Sociedades de beneficio e interés colectivo. [Diapositivas de power point]. https://goo.su/a7Xy

Remacha, M. (2017). Empresa y objetivos de desarrollo sostenible. IESE Business School. Universidad de Navarra. https://goo.su/usY

Real Decreto Legislativo 1/2010, de 2 de julio. (2010). Por el que se aprueba el texto refundido de la Ley de Sociedades de Capital. 3 de julio de 2010. BOE-A-2010-10544 https://goo.su/9OS6

Real Decreto 877/2015. (2015). De desarrollo de la Ley 26/2013, de 27 de diciembre, de cajas de ahorros y fundaciones bancarias, por el que se regula el fondo de reserva que deben constituir determinadas fundaciones bancarias... 2 de octubre de 2015. España. https://goo.su/ahmL

Rubio-Rodríguez, G. y Varón Romero, A. (2018). Valor que subroga el salario emocional como práctica de responsabilidad social empresarial interna en la gobernanza cooperativa. Revista Iberoamericana de Economía Solidaria e Innovación Socioecológica, 1(2018), pp. 87-100.

Salmeron, P. (2019). Análisis de la Certificación B Corp. [Tesis para optar el grado en administración y dirección de empresas. Universidad Politécnica de Cartagena]. https://goo.su/9S8G

Sistema B. (2020). La Asamblea Nacional de Ecuador aprobó por unanimidad la Ley de Emprendimiento e Innovación que reconoce a las Sociedades BIC (Beneficio e Interés Colectivo). https://goo.su/akrQ

Sistema B. (2021). América Central y Caribe. Conoce a las empresas B de América Central. https://goo.su/JOJ 
Sistema B. (2021). Directorio de Empresas B en América Latina y Caribe. https://www.sistemab.org/directorio-b/ pachenge/7/\#main-search

Superintendencia de Sociedades. (2019). Oficio 220-013670 del 28 de febrero de 2019. https://goo.su/9sum

Sustainable Prosperity. (2012). Beneficial corporations. Economy-Wide and emerging issues. https://goo.su/9l6t

Tuchin, F. (25 de mayo de 2021). Ley BIC: ¿por qué necesitamos una legislación que reconozca el triple impacto?. Redacción Periodismo Humano. https://goo.su/9Mgk

Ü, E. (2013). Raising dough: the complete guide to financing a socially responsible food business. Chelsea Green Publishing.

Vilchez, J. (2019). La necesidad de regular las empresas B en el Perú. [Tesis para optar el grado de abogado. Universidad Nacional Pedro Ruiz Gallo]. https://goo.su/9NdB 